\title{
FROM LBV BINARY TO WR+OB BINARY.
}

\author{
D. VANBEVEREN \\ Dept. of Physics, VUB, \\ Brussels, Belgium.
}

SUMMARY. The LBV phase is generally identified with the hydrogen shell burning phase of a star with initial mass larger than $40-50 \mathrm{M}_{\Theta}$ (see also Humphreys, this volume) and therefore in binaries with ZAMS component masses larger than $40-50 \mathrm{M}_{\odot}$ and periods larger than 4-7 days the primary may experience a LBV mass loss phase before it reaches its critical equipotential surface (the Roche lobe). I then define the 'LBV scenario' of massive close binaries as follows:

when a binary component (initial mass larger than 40-50 $\mathrm{M}_{\Theta}$ ) reaches the LBV phase prior to its Roche lobe overflow phase (RLOF), a stellar wind mass loss phase sets in at rates comparable to the rates encounterd during a RLOF process and which are large enough in order to prohibit the occurence of a RLOF.

Evolutionary models are computed for close binaries with initial primary masses larger than $50 \mathrm{Mo}$ and mass ratios ranging between 0.2 and 1 . Special attention is given at the predicted spectral type of the secondary component. In order to determine the mass of the primary at the end of its LBV phase, I have used the following general theorem holding for the most massive stars, i.e.

a hydrogen shell burning mass loser with mass larger than $50 \mathrm{M}_{\odot}$ in a massive close binary restores thermal equilibrium (and becomes a WR star) when helium starts burning in its core and when its atmospherical hydrogen abundance has dropped to $X_{\text {atm }}=0.2-0.3$ (by weight).

Since LBV mass loss is spherically symmetric, the binary period $P$ increases during the LBV phase (i.e. $\mathrm{P} \div\left(\mathrm{M}_{1}+\mathrm{M}_{2}\right)^{-2}, \mathrm{M} 1$ and $\mathrm{M} 2$ are the masses of both components) whereas accretion effects can be neglected (i.e. the mass and spectral type of the companion star remain unchanged during the LBV phase). At the end of the LBV phase the binary consists of a stripped helium core (a WR star) and an OB type giant or supergiant; the binary period should be larger than 10-20 days. Possible WR binary candidates which may have evolved through such a LBV phase are HD 92740, HD 68273, HD 137603 and HD 168206.

This summary is part of a paper which has been submitted to Astron. and Astrophys. 